\title{
Weekend effects of admission and surgery in acute aortic syndrome
}

\author{
Chao Song ${ }^{1}$, YunLong Fan ${ }^{1}$, ShiXiong $\mathrm{Wei}^{1}$, and Shengli Jiang ${ }^{2}$ \\ ${ }^{1}$ Medical School of Chinese PLA \\ ${ }^{2}$ Chinese PLA General Hospital
}

January 8, 2021

\begin{abstract}
Background: The weekend effect is a phenomenon characterized by increased early all-cause mortality among patients hospitalized or undergoing surgery over the weekend for emergencies. Objectives: With this meta-analysis we aimed to determine whether weekend hospitalization/surgery due to acute aortic syndromes (AAS) is associated with increased early all-cause mortality. Methods:Major electronic databases were searched for studies published up to October 2020 reporting early all-cause mortality rates for admissions/operations on weekends versus weekdays. Data were pooled using fixed- and random-effects models. The primary outcome of the study was early all-cause mortality after weekend versus weekday. Results: All the included studies were retrospective, comparative or cohort studies enrolling patients admitted or underwent surgery for AAS and reported early all-cause mortality after weekend (including holiday) versus weekday. A total of 18 studies including a total of 252807 patients were identified. This meta-analysis showed a significant increase in the early all-cause mortality for patients admitted/conducted surgery for AAS on weekends compare with weekdays (fixed-effect: OR 1.1;95\% CI 1.06-1.14; $\mathrm{P} j 0.00001$ ). Conclusion: Weekend admission/surgery for AAS is associated with a increased risk of early all-cause mortality.
\end{abstract}

\section{Hosted file}

Manuscript.pdf available at https://authorea.com/users/388253/articles/503072-weekendeffects-of-admission-and-surgery-in-acute-aortic-syndrome

\section{Hosted file}

04 Table1.pdf available at https://authorea.com/users/388253/articles/503072-weekend-effectsof-admission-and-surgery-in-acute-aortic-syndrome

\section{Hosted file}

05 Table2.pdf available at https://authorea.com/users/388253/articles/503072-weekend-effectsof-admission-and-surgery-in-acute-aortic-syndrome 


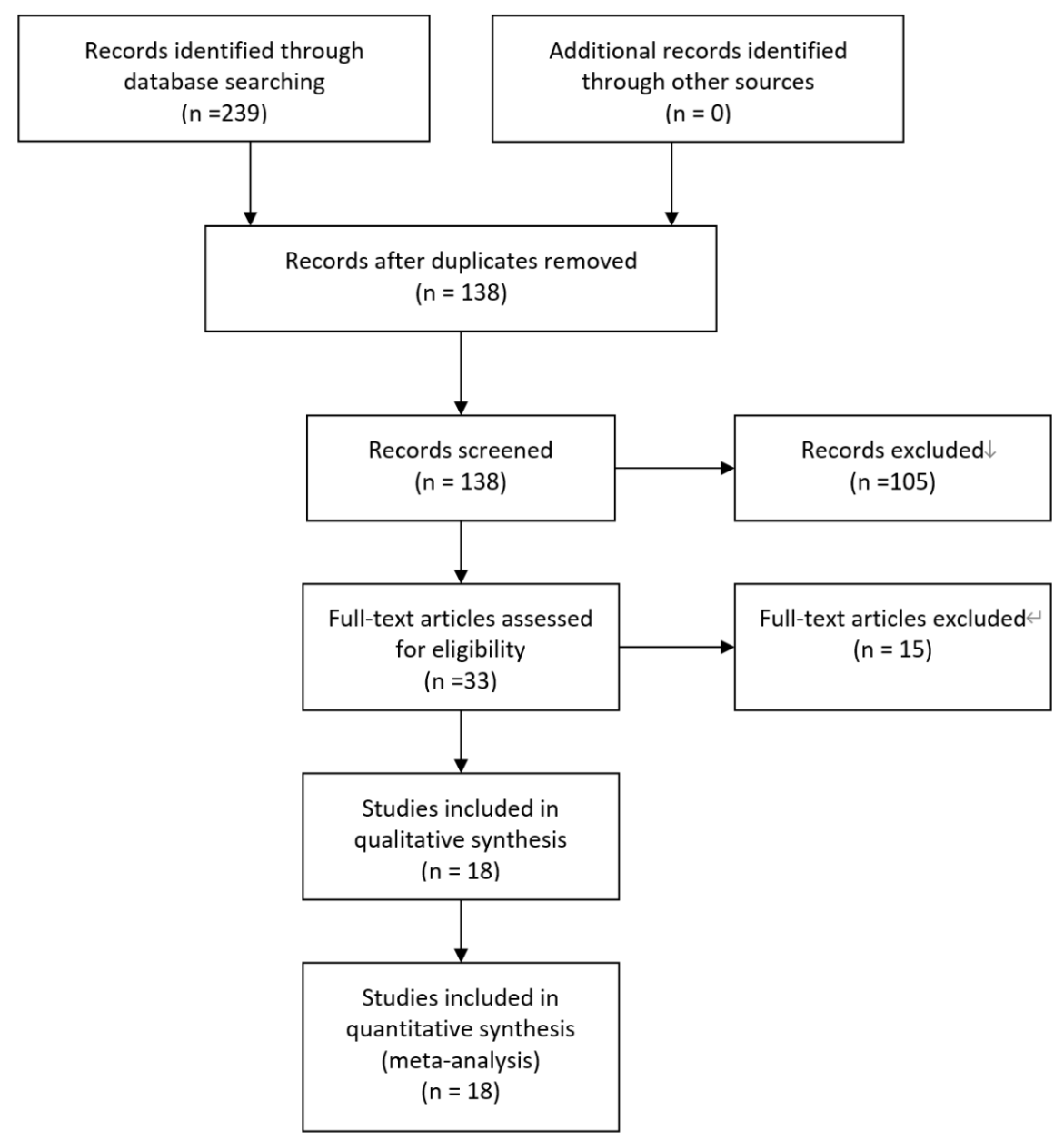

\begin{tabular}{|c|c|c|c|c|c|c|c|}
\hline Studv or Subgroup & log[Odds Ratio] & SE & Weight & $\begin{array}{l}\text { Odds Ratio } \\
\text { IV. Fixed, } 95 \% \mathrm{Cl}\end{array}$ & $\begin{array}{l}\text { Odds } \\
\text { IV, Fixed, }\end{array}$ & $\begin{array}{l}\text { Ratio } \\
\text { d. } 95 \% \mathrm{Cl}\end{array}$ & \\
\hline Ahlsson A 2019 & 0.017033 & 0.237245 & $0.6 \%$ & $1.02[0.64,1.62]$ & & Tt & \\
\hline Alejandro Fierro 2020 & 0.045323 & 0.186224 & $1.0 \%$ & $1.05[0.73,1.51]$ & & 1 & \\
\hline Ambler 2017 & 0.037426 & 0.094388 & $3.8 \%$ & $1.04[0.86,1.25]$ & & $t$ & \\
\hline Ambler GK 2017 & 0.08636 & 0.094388 & $3.8 \%$ & $1.09[0.91,1.31]$ & & - & \\
\hline Behrendt CA 2019 ER & 0.191451 & 0.369643 & $0.2 \%$ & $1.21[0.59,2.50]$ & & & \\
\hline Behrendt CA 2019 OSR & 0.207096 & 0.238265 & $0.6 \%$ & $1.23[0.77,1.96]$ & & + & \\
\hline Bell CM 2001 & 0.10721 & 0.084184 & $4.7 \%$ & $1.11[0.94,1.31]$ & & - & \\
\hline Cram P 2004 & 0.32838 & 0.206633 & $0.8 \%$ & $1.39[0.93,2.08]$ & & & \\
\hline Deshmukh A 2010 & 0.041393 & 0.160714 & $1.3 \%$ & $1.04[0.76,1.43]$ & & 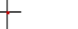 & \\
\hline Gallerani M 2012 & 0.123852 & 0.089286 & $4.2 \%$ & $1.13[0.95,1.35]$ & & - & \\
\hline Gallerani M 2013 & 0.127105 & 0.05102 & $12.9 \%$ & $1.14[1.03,1.25]$ & & $=$ & \\
\hline Groves 2014 & 0.40654 & 0.487245 & $0.1 \%$ & $1.50[0.58,3.90]$ & & & \\
\hline Karthikesalingam 2016 England & 0.025306 & 0.048469 & $14.3 \%$ & $1.03[0.93,1.13]$ & & & \\
\hline Karthikesalingam 2016 Sweden & 0.143015 & 0.221939 & $0.7 \%$ & $1.15[0.75,1.78]$ & & & \\
\hline Karthikesalingam A 2014 England & 0.058426 & 0.057653 & $10.1 \%$ & $1.06[0.95,1.19]$ & & 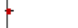 & \\
\hline Karthikesalingam A 2014 US & 0.062958 & 0.084694 & $4.7 \%$ & $1.06[0.90,1.26]$ & & & \\
\hline Kumar N 2016 & 0.068186 & 0.056122 & $10.6 \%$ & $1.07[0.96,1.20]$ & & & \\
\hline Kózka MA 2014 & 0.247973 & 0.362245 & $0.3 \%$ & $1.28[0.63,2.61]$ & & & \\
\hline Ozdemir 2015 & 0.068186 & 0.063776 & $8.2 \%$ & $1.07[0.94,1.21]$ & & & \\
\hline P Aylin 2013 & 0.324282 & 0.625 & $0.1 \%$ & $1.38[0.41,4.71]$ & & & \\
\hline Roberts SE 2019 England & 0.178977 & 0.045153 & $16.4 \%$ & $1.20[1.09,1.31]$ & & & \\
\hline Roberts SE 2019 Wales & 0.28892 & 0.227551 & $0.6 \%$ & $1.33[0.85,2.09]$ & & & \\
\hline Total $(95 \% \mathrm{Cl})$ & & & $100.0 \%$ & $1.10[1.06,1.14]$ & & & \\
\hline \multicolumn{5}{|c|}{$\begin{array}{l}\text { Heterogeneity: } C \mathrm{Ch}^{2}=10.73, \mathrm{df}=21(P=0.97) ; \mathrm{l}^{2}=0 \% \\
\text { Test for overall effect: } Z=5.23(P<0.00001)\end{array}$} & $\begin{array}{ccc}0.01 & 0.1 \\
\text { Favours [weekends] }\end{array}$ & 1 Favours & $\begin{array}{ll}1 & 1 \\
10 & 100 \\
\text { s [weekdays] }\end{array}$ \\
\hline
\end{tabular}




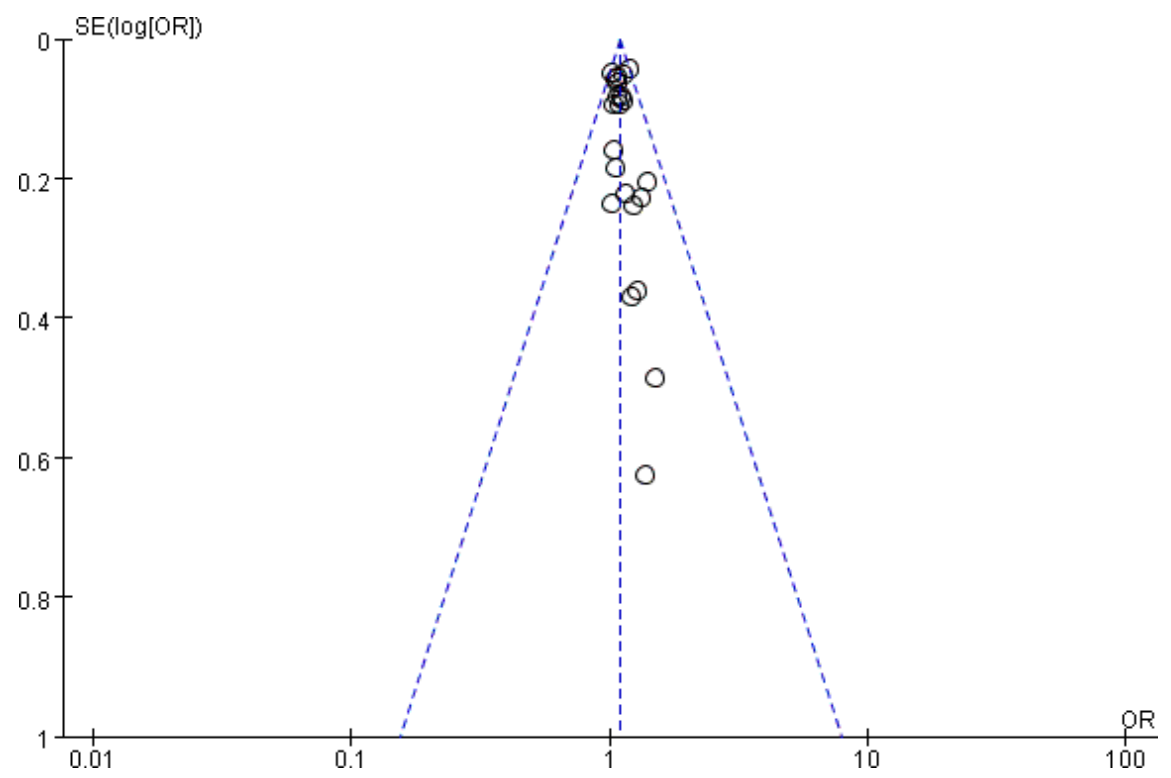

